\title{
Characterising the Radio Frequency Plasma Source for Glow Discharge Optical Emission Spectroscopy
}

\author{
Richard PAYLING, Patrick CHAPON ${ }^{1)}$, Olivier BONNOT ${ }^{1)}$, Philippe BELENGUER ${ }^{2)}$, Philippe GUILLOT ${ }^{2)}$, \\ Leanne C. PITCHFORD ${ }^{2}$, Laurent THERESE ${ }^{2)}$, Johann MICHLER ${ }^{3)}$ and Max AEBERHARD
}

Department of Physics, University of Newcastle, NSW 2308, Australia

1) Jobin-Yvon Emission Horiba Group, 16-18 rue du Canal, 91165 Longjumeau, France

2) CPAT, UMR 5002, 118 route de Narbonne, 31062 Toulouse, France

3) Swiss Federal Laboratories for Materials Testing and Research (EMPA), Feuerwerkerstrasse 39, CH-3602

Thun, Switzerland

The radio frequency (RF) powered source is the most stable and capable analytical source available for glow discharge optical emission spectroscopy (GD-OES). It allows the direct analysis of both conductive and nonconductive samples on the same source, without changing the source. Recent developments in RF generators and components have further improved the performance of these sources, and now allow the routine measurement of additional RF parameters such as applied voltage and DC bias voltage. Theoretical studies of the fundamental characteristics of the RF source are providing deeper understanding of how they work, and combined with the new measurement tools will lead to further improvements in analytical performance. Recent theoretical and experimental work on the RF plasma source will be presented. These studies will be illustrated with some recent applications: in metals analysis, plus a hard coating and a non-conductive coating.

KEY WORDS: radio frequency; glow discharge; GD-OES; plasma sources; analysis; metallic coatings; hard coatings; non-conductive.

\section{Introduction}

Radio frequency powered glow discharge optical emission spectroscopy (RF-GD-OES) is a rapid technique for the bulk analysis and qualitative and quantitative depth profiling of a wide range of conductive and non-conductive materials and coatings.)

In the RF source commonly used in GD-OES, the driven electrode (called cathode) is the sample to be analysed. The cathode and the grounded electrode (anode) are very different in size; see Fig. 1, where $S_{0}$ and $S_{R F}$ are the surface areas of the anode and cathode, respectively.

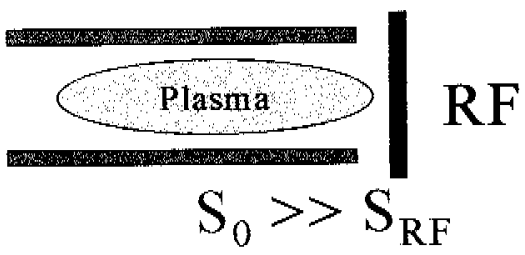

Fig. 1 RF GD-OES source

When a sinusoidal RF voltage is applied to the sample the difference in area of the two electrodes leads to a $\mathrm{DC}$ bias voltage $\left(V_{D C}\right)$ on the sample surface, see Fig. 2, where the applied voltage shown is $1600 \mathrm{~V}$ peak-to-peak, and $V_{D C}=-700 \mathrm{~V}$. During most of the cycle, the sample surface is biased negatively and is only positive for a very short time. No sputtering of the anode occurs during this brief positive phase.

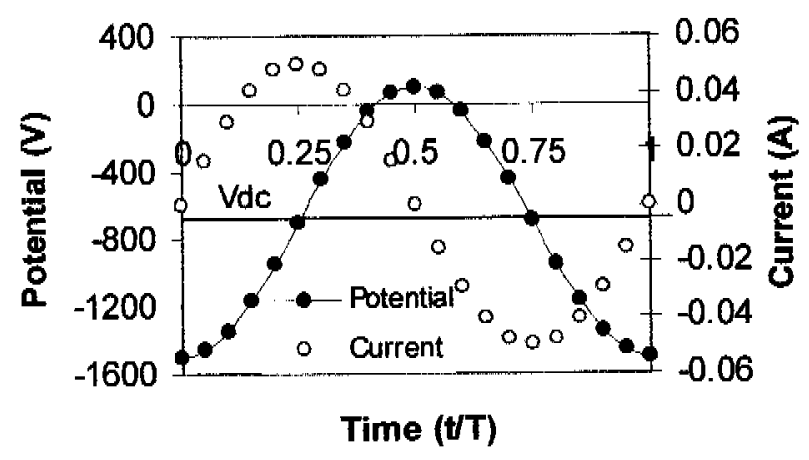

Fig. 2 Idealised Voltage and Current at the Cathode

Because of the capacitive nature of the resulting discharge, the voltage and current waveforms differ in phase. Most of the measured current is displacement current, i.e. due to capacitive coupling and not to electrons flowing in the plasma. Once this displacement current is removed, it is then possible to see the ion and electron currents in the plasma, see Fig. $3{ }^{2}$ During most of the cycle the current is dominated by ion current and only for a very short time by electron current.

Because of its ability to analyse both conductive and nonconductive samples, RF-GD-OES continues to attract widespread research interest, both theoretical and experimental. Besides the scientific interest of better 
understanding the physics of the RF plasma, a principal aim is improved performance and accuracy of the technique. For this reason, we describe recent results both on fundamental studies of the RF source and on instrument performance and applications.

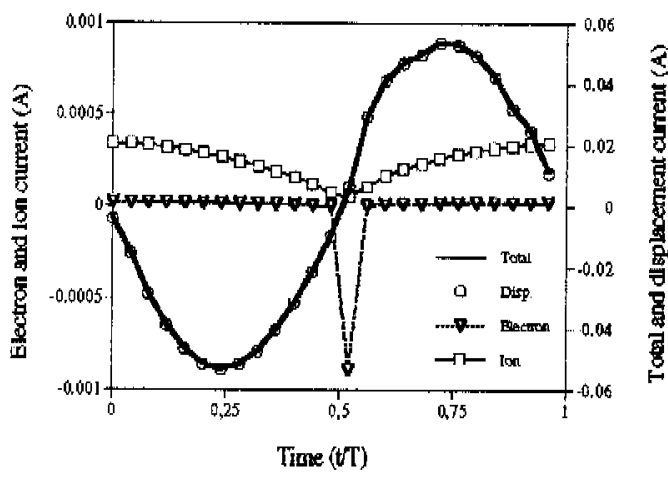

Fig. 3 Idealised Ion and Electron Currents

While an active circuit design is available, the most common way to apply the RF voltage to the sample is through a matching box, see Fig. 4. The impedance of the source may change because of changing source parameters, such as pressure or power, or the varying electron emission characteristics of different samples or different layers on a single sample. By varying the capacitors $\mathrm{Cl}$ and $\mathrm{C} 2$ automatically, the matching box ensures the impedance seen by the RF generator supplying V1 is always $50 . \Omega$. Crucial to the performance of this circuit is the quality of the signal coming from the RF generator. Recent improvements in RF generators have minimised harmonics and allowed precise estimation of reflected power to optimise the settings of $\mathrm{Cl}$ and $\mathrm{C} 2$. The new Class $\mathrm{E}$ generators, for example, have less than $1 \%$ harmonics. This improvement has allowed the analysis of relatively thick glasses and ceramics, up to $5 \mathrm{~mm}$ thick, for the first time. Also the use of vacuum capacitors with high quality motors has improved the speed of adjustment, long-term reliability and precision.

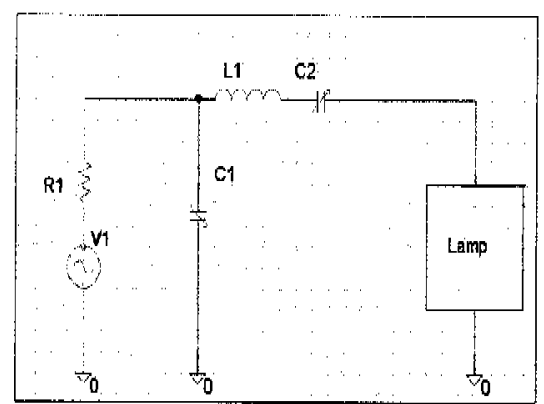

Fig. 4 Schematic diagram of a matching box circuit, showing a fixed inductance $\mathrm{Ll}$ and two variable capacitors $\mathrm{C} 1$ and $\mathrm{C} 2$

\section{Experimental Procedure}

The RF-GD-OES analyses were conducted using two JY 5000 RF instruments (Jobin-Yvon S.A.), or with an identical RF source removed from the instrument to facilitate more fundamental studies of the source. All three sources used $4 \mathrm{~mm}$ anodes. The full instruments were equipped with two optical spectrometers: a polychromator and a monochromator, and Quantum 2000 V1.1 software. All sources were operated with constant pressure monitored in the source and constant applied power from the RF generator. For bulk analysis the source conditions were chosen to optimise signal strength and were typically $900 \mathrm{~Pa}$ and $60 \mathrm{~W}$. The conditions for depth profiling were chosen to give good crater shape and were typically $600 \mathrm{~Pa}$ and $35 \mathrm{~W}$. All three sources were equipped with the new JY matching box that allows continuous monitoring of the DC bias voltage.

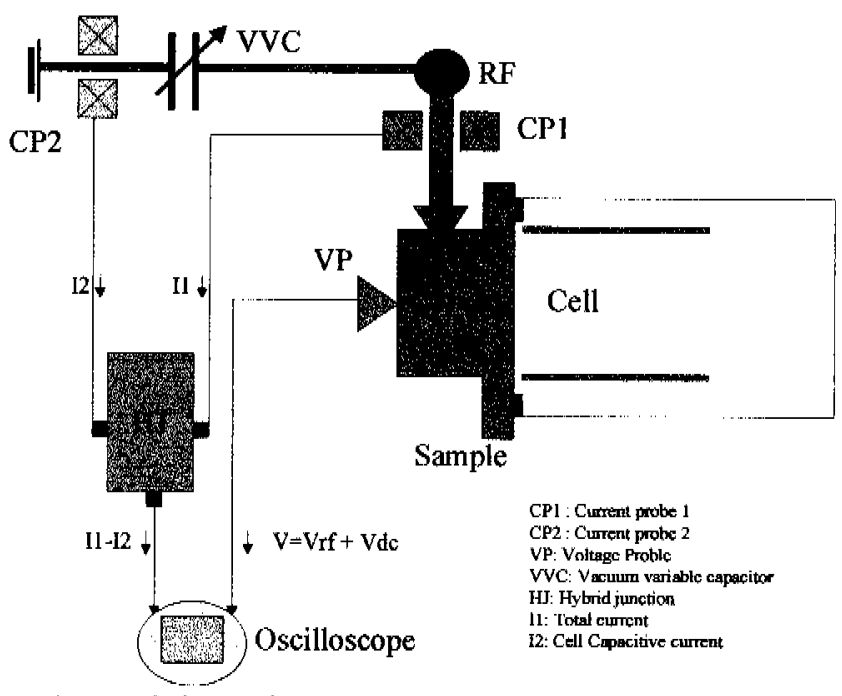

Fig. 5 Schematic diagram of set-up for monitoring RF current and voltage, showing two current probes CP1 and $\mathrm{CP} 2$, voltage probe VP, hybrid junction $\mathrm{HJ}$, oscilloscope, variable capacitor VVC

To monitor the current and voltage characteristics of the isolated source, two currents probes and a voltage probe were used as shown in Fig. 5. Two current probes are needed to subtract the displacement current from the total current to obtain the plasma current. The subtraction was achieved through a hybrid junction and the results seen on a high performance oscilloscope, TDS 3034 . The voltage probe was a Tektronix P.6015 1000x, with $3 \mathrm{pF}$ capacitance and bandwidth of $75 \mathrm{MHz}$. The current probes were Solar 9323-1.

The current probe is a Rogowsky type. The windings of the probe are essentially the secondary windings of a toroidal transformer. The RF cable, carrying the current to be measured, acts as the primary winding of the transformer.

A method for suppressing the displacement current in a GD-OES instrument was developed recently by Hoffmann's laboratory ${ }^{3)}$ but to avoid the complexity of their approach we have used the technique perfected on the GEC (Gaseous Electronics Conference) reference Cell. ${ }^{4)}$.

As in the GEC reference cell, we have used a shunt capacitance (see VVC in Fig. 5) to eliminate the capacitive reactance of the cell. Without plasma gas, the total current is equal to the displacement current, and the variable 
capacitance is adjusted until there is no RF current. When plasma gas is then admitted into the cell the measured current will be just the plasma current without the displacement current.

\section{Theory}

It is common for complex plasma, such as a glow discharge, to use hybrid models, i.e. different models are chosen best suited to different aspects of the plasma and then these models are combined by transferring data backwards and forwards between them. For the study described here we used a self-consistent, two-dimensional hybrid fluid-particle model. ${ }^{5)}$ In this model, fluid equations are used to describe the ions and electrons that contribute to the space charge and these equations are solved selfconsistently with Poisson's equation for the electric field. The model calculates the charged particle densities and the electric field as functions of space and time. We assume cylindrical symmetry and the results thus depend on distances in the radial and axial directions. An important set of input data for this model is the ionisation source terms appearing in the continuity equations for the charged particles. A unique feature of the model used is how these data are determined. A Monte Carlo particle approach is used. A particle treatment of the ionisation source term is necessary because the ionisation source terms in the rapidly varying electric field in the cathode fall of an RF glow discharge are very non-local. ${ }^{6}$

In de discharges, electrons emitted from the cathode and accelerated in the cathode sheath deposit their energy in excitation and ionisation and then diffuse to the anode or sidewalls. When their total energy (kinetic plus potential) drops below the ionisation potential of the neutral gas, the electrons are no longer capable of producing ionisation and they can be dropped from the Monte Carlo simulation. In contrast, in RF discharges, the opposite electrode acts as the cathode for part of the cycle and electrons diffusing to the instantaneous anode can be accelerated in the advancing sheath as the electrodes change polarity. Electrons accelerated in this way are called 'wave-riding' electrons. To include the ionisation produced by these electrons in our Monte Carlo calculation, electrons are included in the simulation all the way until they reach an electrode, i.e. they are not dropped from the simulation when their total energy decreases below the ionisation potential, because they may regain energy from the changing electric field.

When an RF voltage is applied to the powered electrode, even without gas in the cell, a current will develop. This current is a displacement current, resulting from the cell capacitance and is related to the temporal variations of the electric field on the electrode. The displacement current $J_{d}$ for a cell capacitance $C$ is given by

$$
J_{d}=C d V / d t
$$

To see the plasma current, either experimentally or in the theoretical model, we need to subtract this displacement current.

A continuous DC bias voltage is present because of the capacitive coupling of the cell to the exterior circuit. It arises from the difference in surface areas of the two electrodes. During any one RF cycle, the flow of ions and electrons to the two electrodes has to be identical. In a GDOES cell, the surface of the powered electrode ('sample') is much smaller than that of the tubular grounded electrode ('anode'). The DC bias voltage compensates for this difference in surface areas by stabilizing the currents. Because of the very great difference in mass between the ions and electrons in the plasma, and their subsequent difference in inertia to changes in the RF field, the electron current is much higher than the ion current. The DC bias voltage is therefore predominantly a response to the electron current. Electrons arriving at the tubular anode will leave to ground, while electrons arriving at the sample cannot flow away because of the blocking capacitor (see C2 in Fig. 4). The sample surface therefore gains a negative potential. During each cycle, the phenomenon reproduces itself to equalise the currents to the two electrodes.

Since the DC bias voltage is compensating for the electron current it should therefore vary with the secondary electron emission yield $\gamma$ (the average number of electrons emitted per incident ion) of the powered electrode. Semiempirically it has been found that $\gamma$ varies inversely to the work function, i.e. ${ }^{7,8)}$

$$
\gamma=0.032\left(0.78 E_{i}-2 \phi\right)
$$

where $E_{j}$ is the effective potential energy (assumed to be the argon ionisation energy, $15.76 \mathrm{eV}$ ).

\section{Results}

\subsection{Model: Theory}

The gas considered in the model calculations was argon at 1.5 Torr and $300 \mathrm{~K}$. Note it is gas density that is important in the model and these conditions are equivalent to an argon pressure of 4.5 Torr at $900 \mathrm{~K}$, typical of a GD plasma. The grounded electrode was $4 \mathrm{~mm}$ diameter. The applied voltage was sinusoidal, i.e. $V=V r f \cos (\omega t)$, with Vrf $=-800 \mathrm{~V}$, and the frequency of the applied voltage either $4 \mathrm{MHz}$ or $13.56 \mathrm{MHz}$, to see the effect of varying the frequency.

Under these conditions, the model yields a value for $V_{d c}$ of $-725 \mathrm{~V}$, and the sheath potential of the grounded electrode ('anode') never exceeds $75 \mathrm{~V}$. Because of the large DC bias, the powered electrode (sample) acts as a cathode during most part of the cycle. Hence, as in a DC plasma, the powered electrode is responsible for the major part of the ionisation mechanisms. The model also suggests that at the conditions chosen, $V_{\mathrm{dc}}$ does not depend on the applied frequency.

At $13.56 \mathrm{MHz}$, the calculated peak displacement current was $5 \mathrm{~mA}$. In the model, the powered electrode surface was about $0.12 \mathrm{~cm}^{-2}$, so the current density for the displacement current was $40 \mathrm{~mA} \mathrm{~cm}{ }^{-2}$, a high value. The displacement current at $4 \mathrm{MHz}$ was about one-third this value.

\subsection{Model: Experiment}

An important step in removing the displacement current is knowing the typical range of cell capacitances 
experienced in GD-OES. We measured this capacitance by applying an RF potential to the cell without plasma gas. Figs. 6 shows the applied voltage and the measured current for a steel sample. Both voltage and current were sinusoidal and differed in phase by $-90^{\circ}$. The cell is therefore purely capacitive. The model calculations are in qualitative agreement with the experimental values, see Fig. 7.

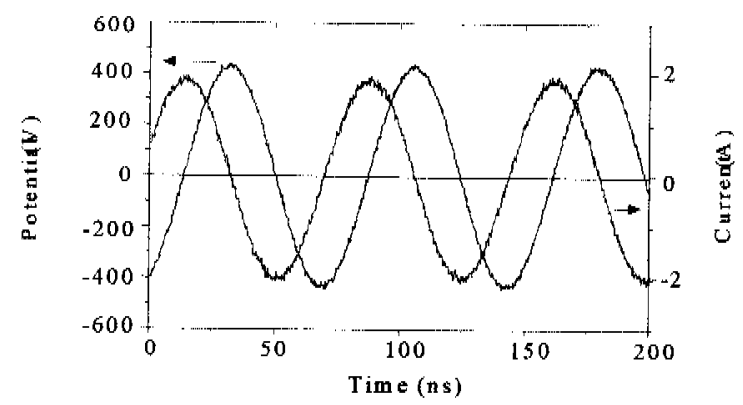

Fig. 6 Measured RF potential and current, for a steel sample: applied power $10 \mathrm{~W}$, no plasma gas

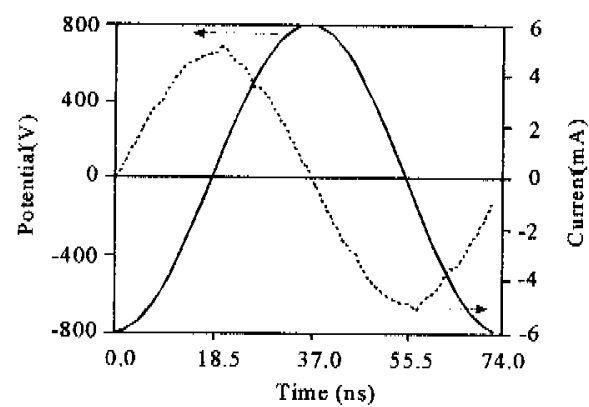

Fig. 7 Calculated RF potential and total current during one cycle: $\mathrm{V}_{\mathrm{RF}}=800 \mathrm{~V}$, no plasma gas

The cell capacitance was calculated using equation (1) for different sample types and different applied powers. Some of the values obtained are shown in Table 1. For conducting samples the cell capacitance was about $30 \mathrm{pF}$, and for non-conducting samples it was about $26 \mathrm{pF}$. This small difference is due to the effect of the capacitance of the non-conducting samples on the total impedance of the cell:

$\frac{1}{C_{\text {total }}}=\frac{1}{C_{\text {cell }}}+\frac{1}{C_{\text {sample }}} \Rightarrow C_{\text {total }}=\frac{C_{\text {cell }} \times C_{\text {sample }}}{C_{\text {cell }}+C_{\text {sample }}}$

This suggests the capacitance of the non-conductive samples was of the order of $200 \mathrm{pF}$.

Table 1. Variation in cell capacitance

\begin{tabular}{lcc}
\hline Sample & $\begin{array}{c}\text { Incident Power } \\
(\mathrm{W})\end{array}$ & $\begin{array}{c}\text { Cell Capacitance } \\
(\mathrm{pF})\end{array}$ \\
\hline Steel & 10 & 29 \\
Steel & 20 & 31 \\
Glass & 10 & 26 \\
Ceramic & 30 & 25 \\
\hline
\end{tabular}

Fig. 8 shows the applied RF voltage and measured total current for a zinc coated steel sample, but with 6 Torr of argon. No shunt capacitor has been used. Both signals remain nearly sinusoidal with a phase difference of nearly $-90^{\circ}$, indicating that the cell impedance is still mostly capacitive. When the RF voltage signals are compared, no DC bias is evident in Fig. 6 in the absence of argon, but in Fig. 8 a DC bias of $-300 \mathrm{~V}$ is present, resulting from the argon plasma. In Fig. 8, the potential oscillates between $-600 \mathrm{~V}$ and $+50 \mathrm{~V}$, but is negative during most of the cycle. The total current is about $3 \mathrm{~A}$, which is very high considering the total current without argon was of the order of 10 to $100 \mathrm{~mA}$. The total current is dominated by the displacement current. The plasma current can be seen in Fig. 8 as small perturbations on the sinusoidal displacement current.

The DC bias voltage was measured for a range of pure metals, at a constant $50 \mathrm{~W}$ and $600 \mathrm{~Pa}$. The results are shown in Fig. 9 and support the hypothesis discussed earlier that the DC bias increases linearly with work function.

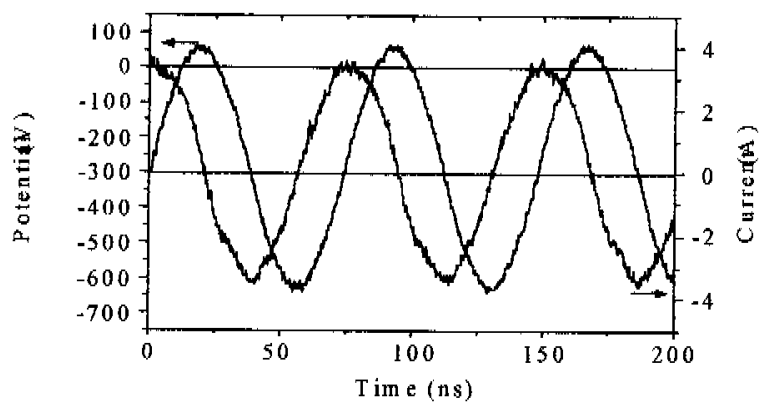

Fig. 8 Measured RF potential and current, for a zinc coated steel sample: applied power $10 \mathrm{~W}, 6$ Torr

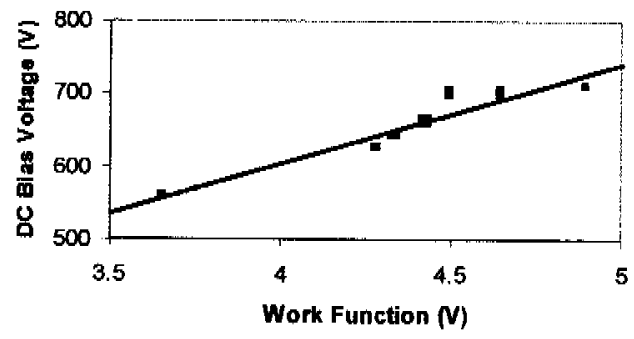

Fig. 9 DC bias voltage for pure metals versus work function

\subsection{Applications}

The first step in analysis is to ensure the instrument has good stability and precision. Assuming good calibration with sufficient number of calibration samples, the relative accuracy should then be similar to the relative precision. $\left.{ }^{9}\right)$ With the improvements to the RF generator and matching box, described earlier, the precision is now typically $0.3 \%$ for flat, clean, homogeneous metal samples. The accuracy obtained on similar certified reference materials (CRMs) is then comparable to the accuracy of the certified concentrations of those CRMs, as shown in Table 2 for a stainless steel CRM No. 465/1. The standard deviation (SD) gives an estimate of the precision of the measurement and comparison of the measured concentration and the certified concentration gives an estimate of the accuracy. 
Table 2. RF-GD-OES Analysis of Stainless Steel 465/1

\begin{tabular}{lccc}
\hline Element & $\begin{array}{c}\text { Certified } \\
\text { (mass \%) }\end{array}$ & $\begin{array}{c}\text { Measured } \\
\text { (mass \%) }\end{array}$ & $\begin{array}{c}\text { SD } \\
\text { (mass \%) }\end{array}$ \\
\hline $\mathrm{Fe}$ & 70.8 & 71.3 & 0.01 \\
$\mathrm{Cr}$ & 17.3 & 17.5 & 0.05 \\
$\mathrm{Ni}$ & 9.2 & 9.1 & 0.04 \\
$\mathrm{Mn}$ & 1.38 & 1.38 & 0.01 \\
$\mathrm{Si}$ & 0.40 & 0.39 & 0.01 \\
$\mathrm{Cu}$ & 0.098 & 0.091 & 0.008 \\
$\mathrm{Mo}$ & 0.092 & 0.087 & 0.004 \\
$\mathrm{C}$ & 0.066 & 0.066 & 0.003 \\
$\mathrm{P}$ & 0.021 & 0.021 & 0.002 \\
$\mathrm{~S}$ & 0.012 & 0.012 & 0.001 \\
\hline
\end{tabular}

One of the longer-term aims of GD-OES analysis is to be able to analyse any coating of any composition with a single calibration. We are working towards this. The first step is to combine many different metal matrices in a single calibration. This can be achieved with RF-GD-OES by including a DC bias correction. The details are explained in ref. 10. A calibration, for example, for $\mathrm{Zn} / \mathrm{Al}$ coatings on steel typically includes $\mathrm{Zn} / \mathrm{Al}$ alloys, brass, and stainless steel samples. Such calibration curves can then be used to obtain bulk analysis or quantitative depth profiles of any metal or metallic coating falling within the wide range of the calibration curves. An example of a quantitative depth profile of galvanised steel is shown in Fig. 10.

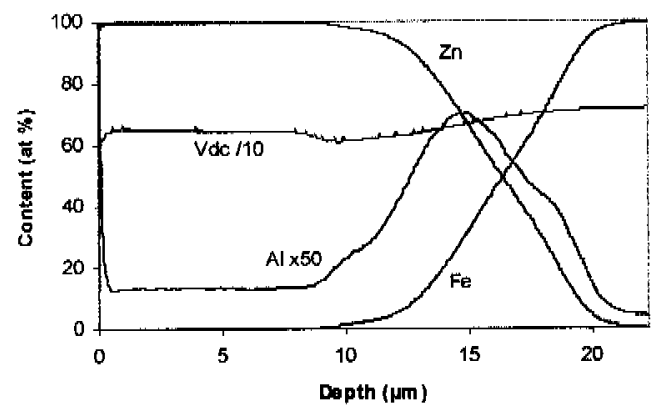

Fig. 10 Galvanised steel.

The calibration for hard coatings typically includes the range of metal alloys used for the $\mathrm{Zn} / \mathrm{Al}$ coated steel plus specially prepared reference hard coatings and CRMs high in $\mathrm{N}$ and $\mathrm{O}$. An example of a quantitative depth profile of TiMoN coated steel is shown in Fig. 11.

It is not possible at present to measure routinely the $\mathrm{DC}$ bias voltage on a non-conducting sample. This is because the DC bias for such a sample is formed only on the inside surface of the sample facing the plasma. Quantitative depth profiling of non-conductive coatings has been possible since 1993 when Payling and Jones reported results on prepainted steel. ${ }^{11)}$ Their approach is called 'matrix-matching', i.e., calibration samples are chosen so that they have similar matrices to the unknown samples. This ensures the emission yields are constant between calibration and analysis. This is the only successful approach for non-conductive samples to date with GD-OES. A recent example of this approach is shown in Fig. 12, a $\mathrm{ZrOx}-\mathrm{YOx}$ plasma sprayed coating on steel. The analysis of non-conductive coatings and bulk samples is an expanding area of interest for GD-OES and it is hoped the ability to measure more RF plasma parameters will aid developments in this work.

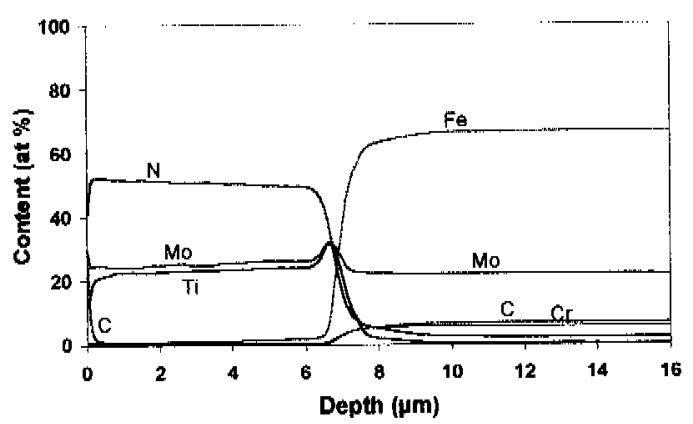

Fig. 11 TiMoN coated steel.

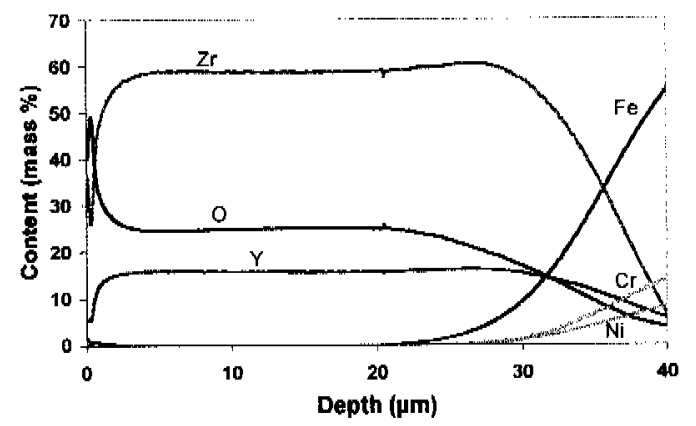

Fig. 12 ZrOx-YOx plasma coating on High Alloy Steel

\section{Conclusions}

Recent developments in RF-GD-OES have increased our understanding of how the RF technique works and improved the performance of the technique, in terms of precision, calibration ranges, and breadth of applications for quantitative depth profiling. Future work will continue this close relationship between RF theory and instrument development.

\section{REFERENCES}

1) R. Payling: Spectroscopy, 13 (1999), 26.

2) P. Chapon, et al:: Patent pending (2000).

3) L. Wilken, V. Hoffmann and K. Wetzig, Poster TP $24,10^{\text {th }}$ BNASS, 17-20 July 2000, Sheffield, U.K. (2000).

4) P. J. Hargis: Rev. Sci. Instrum., 65 (1994), 140.

5) A. Fiala, L. C. Pitchford and J. P. Boeuf: Phys. Rev. E, 49 (1994), 5607.

6) J. P. Bceuf and E. Marode: J. Phys. D, 15 (1982), 2169.

7) R. A. Baragiola, E. V. Alonso, J. Ferron and A. Oliva Florio: Surf. Sci., 90 (1979), 915.

8) L. M. Kishinevsky: Radiation Effects, 19 (1973), 23.

9) R. Payling: Glow Discharge Optical Emission Spectrometry, ed. by R. Payling, D. G. Jones and A. Bengtson, John Wiley \& Sons, Chichester, (1997), 428.

10) R. Payling, M. Aeberhard and D. Delfosse: J. Anal. At. Spectrom., 50 (2001), 16.

11) R. Payling, D. G. Jones and S. A. Gower: Surf. Interface Anal., 20 (1993), 959. 